\title{
Topological Properties of a 3-Regular Small World Network
}

\author{
Huanshen Jia, ${ }^{1}$ Guona Hu, ${ }^{1}$ and Haixing $\mathrm{Zhao}^{2}$ \\ ${ }^{1}$ Department of Mathematics, Qinghai Normal University, Xining 810008, China \\ ${ }^{2}$ Department of Computer Science, Qinghai Normal University, Xining 810008, China
}

Correspondence should be addressed to Haixing Zhao; h.x.zhao@163.com

Received 9 February 2014; Revised 11 April 2014; Accepted 12 April 2014; Published 29 April 2014

Academic Editor: Jinde Cao

Copyright (C) 2014 Huanshen Jia et al. This is an open access article distributed under the Creative Commons Attribution License, which permits unrestricted use, distribution, and reproduction in any medium, provided the original work is properly cited.

\begin{abstract}
Complex networks have seen much interest from all research fields and have found many potential applications in a variety of areas including natural, social, biological, and engineering technology. The deterministic models for complex networks play an indispensable role in the field of network model. The construction of a network model in a deterministic way not only has important theoretical significance, but also has potential application value. In this paper, we present a class of 3-regular network model with small world phenomenon. We determine its relevant topological characteristics, such as diameter and clustering coefficient. We also give a calculation method of number of spanning trees in the 3-regular network and derive the number and entropy of spanning trees, respectively.
\end{abstract}

\section{Introduction}

In recent years, research on complex networks is in the ascendant. Complex networks by using graph theory and some methods of statistical physics can be used to capture and describe the evolution of the system mechanism, evolution pattern, and the overall behavior, which is one of the main causes of vigorous development on complex networks research [1].

Construction of small-world networks that conform to the real system features not only has important theoretical significance but also has potential application value in deterministic way [2]. In this paper, we present 3-regular small-world network. We determine the relevant topological characteristics of the regular network, such as diameter and clustering coefficient. The results show that our model has a discrete exponential degree distribution, high clustering, and small diameter, which appears in a small-world effect. It is known that the number of spanning trees is an important quantity characterizing the reliability of a network. Because of the diverse applications in a number of fields, a lot of efforts have been devoted to the study of spanning trees. For example, in $[3,4]$ the author gave the accurate number of spanning trees in regular lattices. The number of spanning trees in a network can be obtained by directly calculating a related determinant corresponding to the network. We also give a method to calculate the number of spanning trees of the 3-regular network and derive the formulas. Based on the number of spanning trees we determine the entropy of its spanning trees.

\section{Network Construction}

The degree $d(v)$ of a vertex $v$ in $G$ is the number of edges of $G$ incident with $v$, each loop counting as two edges [5]. A graph $G$ is $k$-regular if $d(v)=k$ for all $v \in V$. In this section we describe a model of growing network, which is constructed in an iterative manner. We denote our network after $t$ steps by $U_{t}(t \geq 1)$. Then the network is constructed in the following way. For $t=1, U_{1}$ is a graph that two nodes connect three edges. For $t \geq 2, U_{t}$ is obtained from $U_{t-1}$ by adding two new nodes at step $t=1$; then two edges are increased; replicate it and connect four new nodes. The process is repeated till the desired graph order is reached; see Figure 1.

Now we compute the size and order of $U_{t}$. Denote by $n_{v}(t)$ the total number of nodes in the network and by $n_{e}(v)$ the total number of edges in the network. It is easy to get that

$$
\Delta n_{v}(t)=n_{v}(t)-n_{v}(t-1)=2 \Delta n_{v}(t-1), \quad t>1 .
$$



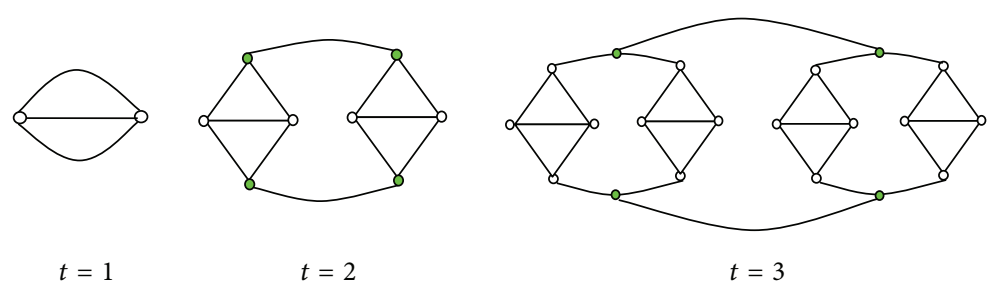

FIGURE 1: Construction of the deterministic 3-regular network $U_{t}$, showing three steps of the iterative progress.

Since $n_{v}(1)=2$ and $\Delta n_{v}(2)=6$, it follows that

$$
\Delta n_{v}(t)=3 \times 2^{t-1}, \quad n_{v}(t)=3 \times 2^{t}-4 .
$$

Note that the addition of four new nodes leads to two new edges. Then

$$
\Delta n_{e}(t)=n_{e}(t)-n_{e}(t-1)=3 \Delta n_{v}(t-1)=9 \times 2^{t-2} .
$$

As $n_{e}(1)=3$, we have

$$
n_{e}(t)=9 \times 2^{t-1}-6 .
$$

\section{Relevant Characteristics}

Due to the determinacy, the relevant characteristics of our model described above can be solved exactly. In the following we concentrate on the diameter and clustering coefficient.

3.1. Diameter. Small-world networks describe many real-life networks; that is, there is a relatively short distance between most pairs of nodes in most real-life networks and their average path length (APL) does not increase linearly with the system size but grows logarithmically with the number of nodes or slower. The average path length is the smallest number of links connecting a pair of nodes, averaged over all pairs of nodes [2]. The longest shortest path between all pairs of nodes is called diameter, which is one of the most important evaluation index because it characterizes the maximum communication delay in the network [6]. For most network models, it is hard to obtain the analytic solution of the average path length. So we will study the diameter instead of average path length. We denote the diameter at iteration $t$ as $D(t)$; then according to Figure 1, we can clearly know that $D(1)=1$ and $D(2)=3$. At each iteration for $t>2$, one can see that the diameter always lies between a pair of newly created nodes at this iteration. Thus the diameter for the network proposed has the following formula:

$$
D(t)=2 t-1=\frac{\ln N_{t}}{\ln 2} .
$$

So, the diameter $D(t)$ grows logarithmically with the number of nodes. Because the average path length is smaller than diameter, the APL should increase more slowly. So our model satisfies the property for small-world networks.

3.2. Clustering Coefficient. Clustering is another important property of a complex network, which provides a measure of the local structure within the network [2]. The clustering coefficient indicates the connection relationship between a node and its neighborhood nodes. By definition, clustering coefficient $C_{i}$ of a node $i$ with degree $k_{i}$ is the ratio of the total number $e_{i}$ of existing edges between all its $k_{i}$ nearest neighbors and the number $k_{i}\left(k_{i}-1\right) / 2$ of all possible edges between them; that is, $C_{i}=2 e_{i} / k_{i}\left(k_{i}-1\right)$. The clustering coefficient $C$ of the whole network is the average of all individual $C_{i}^{\prime} s$; that is, $C=(1 / N) \sum_{i=1}^{N} C_{i}$, where $N$ is the number of nodes of the network. So the clustering coefficient for our network has the following simple formula. We know $C=1$ at step $t=1$. At each iteration $t \geq 2$, we have

$$
C=\frac{2^{t}}{3 \cdot 2^{t}-4} .
$$

Obviously, $C \approx 1 / 3$ when $t \rightarrow \infty$; thus the clustering is high and also satisfies the properties for small-world networks.

Based on the above discussion, we can conclude that our model is a deterministic small-world network, because it is sparse with small diameter and average path length and high clustering coefficient.

\section{Enumeration of Spanning Trees}

A spanning tree of any connected network is defined as a minimal set of edges that connect every node. The enumeration of spanning trees in networks is a fundamental issue in mathematics [7-9], physics [3, 10], and other disciplines [11]. The problem of spanning trees is relevant to various aspects of networks, such as reliability $[12,13]$, optimal synchronization [14], and random walks [15]. In what follows we will examine the number of spanning trees in the 3-regular network.

In order to calculate the number of spanning trees accurately, at first we build a new model $F_{t}$ shown in Figure 2. We list the relationship between $U_{t}$ and $F_{t}$ in Figure 3. The number of the spanning trees and the spanning forests with two components such as $u$ and $v$ belongs to distinct components of $F_{t}$ that are denoted by $\operatorname{ST}\left(F_{t}\right)$ and $\operatorname{SF}\left(F_{t}\right)$, respectively. Then the number of spanning trees of the original model $U(t)$ can be obtained from the number of the spanning trees of $F_{t}$. Then we have the following relationship:

$$
\operatorname{ST}\left(U_{t}\right)=2 \operatorname{ST}^{2}\left(F_{t-1}\right)+2 \operatorname{ST}\left(F_{t-1}\right) \cdot \operatorname{SF}\left(F_{t-1}\right) .
$$

We calculate the number of spanning trees of $F_{t}$ in the following. It is easy to obtain the relationship of $\operatorname{ST}\left(F_{t}\right)$ and $\mathrm{ST}\left(F_{t-1}\right)$ from (7). From Figure 4, one can see that the shape of the spanning trees of $F_{t}$ includes two cases. 


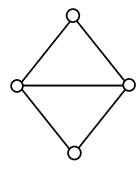

$t=1$

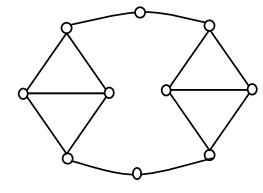

$t=2$

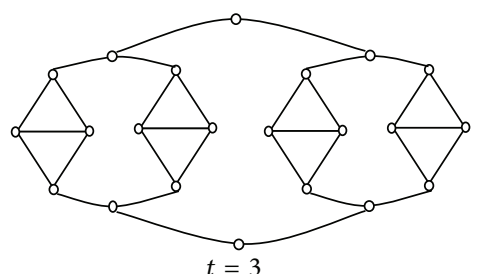

FIGURE 2: Construction of the deterministic 3-regular network $F_{t}$, showing three steps of the iterative progress.
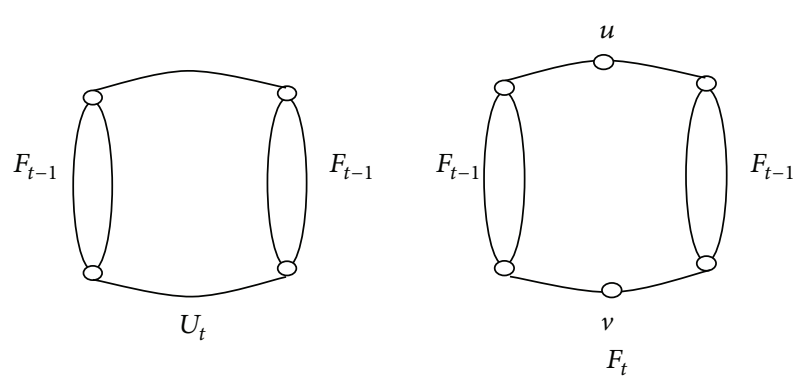

FIGURE 3: The network models $U_{t}$ and $F_{t}$ at step $t$.

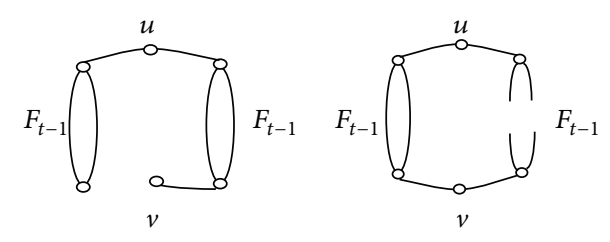

FIGURE 4: The number of spanning trees of $F_{t}$.

And the shape of the spanning forests with two components such as $u$ and $v$ belongs to distinct components of $F_{t}$ that have five cases; see Figure 5.

By considering the symmetric, it is not hard to get the recurrence relation of the number of spanning trees and spanning forests of $F_{t}$ and $F_{t-1}$ in Figures 4 and 5:

$$
\begin{gathered}
\operatorname{ST}\left(F_{t}\right)=4 \mathrm{ST}^{2}\left(F_{t-1}\right)+2 \mathrm{ST}\left(F_{t-1}\right) \cdot \operatorname{SF}\left(F_{t-1}\right), \\
\mathrm{SF}\left(F_{t}\right)=4 \mathrm{ST}^{2}\left(F_{t-1}\right)+\mathrm{SF}^{2}\left(F_{t-1}\right)+4 \operatorname{ST}\left(F_{t-1}\right) \cdot \operatorname{SF}\left(F_{t-1}\right),
\end{gathered}
$$

with the initial conditions $\operatorname{ST}\left(F_{1}\right)=8$ and $\operatorname{SF}\left(F_{1}\right)=8$. By computation, it follows that

$$
\begin{aligned}
& \frac{\operatorname{ST}\left(F_{t}\right)}{\operatorname{SF}\left(F_{t}\right)} \\
& =\frac{4 \cdot\left(\mathrm{ST}^{2}\left(F_{t-1}\right) / \mathrm{SF}^{2}\left(F_{t-1}\right)\right)+2 \cdot\left(\mathrm{ST}\left(F_{t-1}\right) / \mathrm{SF}\left(F_{t-1}\right)\right)}{4 \cdot\left(\mathrm{ST}^{2}\left(F_{t-1}\right) / \mathrm{SF}^{2}\left(F_{t-1}\right)\right)+4 \cdot\left(\operatorname{ST}\left(F_{t-1}\right) / \mathrm{SF}\left(F_{t-1}\right)\right)+1} .
\end{aligned}
$$

Let $a_{t}=\operatorname{ST}\left(F_{t}\right) / \operatorname{SF}\left(F_{t}\right)$; then $a_{t}=2 a_{t-1} /\left(2 a_{t-1}+1\right)$ with $a_{1}=1$. So $a_{t}=2^{t-1} /\left(2^{t}-1\right)$.
Noticing that $\operatorname{SF}\left(F_{t}\right)=\left(\left(2^{t}-1\right) / 2^{t-1}\right) \cdot \operatorname{ST}\left(F_{t}\right)$, by $(8)$, we know that

$$
\begin{gathered}
\operatorname{ST}\left(F_{t}\right)=2^{3 \cdot 2^{t}-3} \cdot \prod_{i=0}^{t-2}\left(1-\frac{1}{2^{t-i}}\right)^{2^{i}}, \\
\operatorname{SF}\left(F_{t}\right)=2^{3 \cdot 2^{t}-2} \cdot\left(1-\frac{1}{2^{t}}\right) \cdot \prod_{i=0}^{t-2}\left(1-\frac{1}{2^{t-i}}\right)^{2^{i}} .
\end{gathered}
$$

Note that $N_{t}^{\prime}=3 \cdot 2^{t}-2$ is the number of nodes of $F_{t}$. From (10) we determine the entropy of the spanning trees of $F_{t}[7,16]$ as follows:

$$
E_{\mathrm{ST}}\left(F_{t}\right)=\lim _{t \rightarrow \infty} \frac{\ln \mathrm{ST}\left(F_{t}\right)}{N_{t}^{\prime}}=\ln 2 \approx 0.6931 .
$$

From (10) and (11), together with (7), the number of spanning trees of $U_{t}$ is given by

$$
\begin{aligned}
\operatorname{ST}\left(U_{t}\right)= & 2 \operatorname{ST}\left(F_{t-1}\right)\left(\operatorname{ST}\left(F_{t-1}\right)+\operatorname{SF}\left(F_{t-1}\right)\right) \\
= & 2^{6 \cdot 2^{t-1}-5} \cdot\left[1+2\left(1-\frac{1}{2^{t-1}}\right)\right] \\
& \times\left[\prod_{i=0}^{t-3}\left(1-\frac{1}{2^{t-i-1}}\right)^{2^{i}}\right]^{2},
\end{aligned}
$$

with the initial condition $\mathrm{ST}\left(U_{1}\right)=1$.

So, the entropy of the spanning trees of $U_{t}$ is given by

$$
E_{\mathrm{ST}}\left(U_{t}\right)=\lim _{t \rightarrow \infty} \frac{\ln \mathrm{ST}\left(U_{t}\right)}{N_{t}}=\ln 2 \approx 0.6931 .
$$

We can compare the entropy of the spanning trees of $U_{t}$ with other networks $[17,18]$. The entropy of the spanning trees in Koch networks with an average degree of 3 is 0.5493 [17], while the entropy of the spanning trees of our model is 0.6931 .

\section{Conclusions}

In this paper, we proposed a class of deterministic regular small-world model which is constructed in an iterative manner and presented an exhaustive analysis of many properties of considered model. Then we obtained the analytic solutions for most of the topological features, including diameter and clustering coefficient. We also determined the number of spanning trees in the 3-regular small-world network. In addition, using the algorithm, we obtained the entropies of spanning trees. 


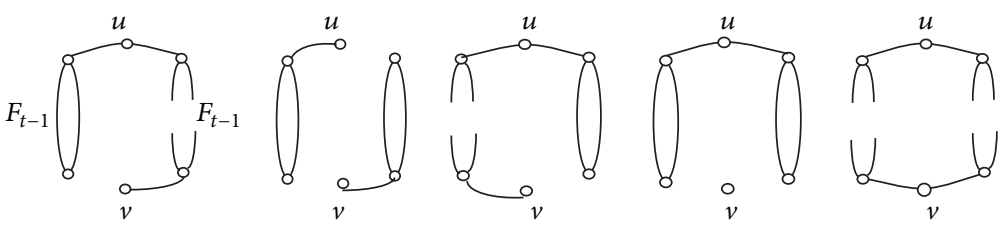

FIGURE 5: The number of spanning forests with two components of $F_{t}$.

\section{Conflict of Interests}

The authors declare that there is no conflict of interests regarding the publication of this paper.

\section{Acknowledgments}

This research is supported by the National Science Foundation of China (nos. 61164005 and 60863006), the National Basic Research Program of China (no. 2010CB334708), and Program for Changjiang Scholars and Innovative Research Team in University (no. IRT1068).

\section{References}

[1] R. Albert and A.-L. Barabási, "Statistical mechanics of complex networks," Reviews of Modern Physics, vol. 74, no. 1, pp. 47-97, 2002.

[2] Z.-Z. Zhang, L.-L. Rong, and F. Comellas, "Evolving smallworld networks with geographical attachment preference," Journal of Physics A: Mathematical and General, vol. 39, no. 13, pp. 3253-3261, 2006.

[3] F. Y. Wu, "Number of spanning trees on a lattice," Journal of Physics A: General Physics, vol. 10, no. 6, Article ID L113, 1977.

[4] R. Shrock and F. Y. Wu, "Spanning trees on graphs and lattices in $\mathrm{d}$ dimensions," Journal of Physics A: Mathematical and General, vol. 33, no. 21, pp. 3881-3902, 2000.

[5] J. A. Bondy and U. S. R. Murty, Graph Theory with Applications, American Elsevier, New York, NY, USA, 1976.

[6] Z.-M. Lu and S.-Z. Guo, "A small-world network derived from the deterministic uniform recursive tree," Physica A: Statistical Mechanics and its Applications, vol. 391, no. 1-2, pp. 87-92, 2012.

[7] R. Burton and R. Pemantle, "Local characteristics, entropy and limit theorems for spanning trees and domino tilings via transfer-impedances," The Annals of Probability, vol. 21, no. 3, pp. 1329-1371, 1993.

[8] R. Lyons, "Asymptotic enumeration of spanning trees," Combinatorics Probability and Computing, vol. 14, no. 4, pp. 491-522, 2005.

[9] R. Lyons, R. Peled, and O. Schramm, "Growth of the number of spanning trees of the Erdős-Rényi giant component," Combinatorics Probability and Computing, vol. 17, no. 5, pp. 711-726, 2008.

[10] S.-C. Chang, L.-C. Chen, and W.-S. Yang, "Spanning trees on the Sierpinski gasket," Journal of Statistical Physics, vol. 126, no. 3, pp. 649-667, 2007.

[11] R. Jayakumar, K. Thulasiraman, and M. N. S. Swamy, "MODCHAR: an implementation of Char's spanning tree enumeration algorithm and its complexity analysis," IEEE Transactions on Circuits and Systems, vol. 36, no. 2, pp. 219-228, 1989.
[12] F. T. Boesch, "On unreliability polynomials and graph connectivity in reliable network synthesis," Journal of Graph Theory, vol. 10, no. 3, pp. 339-352, 1986.

[13] G. J. Szabó, M. Alava, and J. Kertész, "Geometry of minimum spanning trees on scale-free networks," Physica A: Statistical Mechanics and its Applications, vol. 330, no. 1-2, pp. 31-36, 2003.

[14] T. Nishikawa and A. E. Motter, "Synchronization is optimal in nondiagonalizable networks," Physical Review E: Statistical, Nonlinear, and Soft Matter Physics, vol. 73, no. 6, Article ID 065106, 2006.

[15] D. Dhar and A. Dhar, "Distribution of sizes of erased loops for loop-erased random walks," Physical Review E: Statistical Physics, Plasmas, Fluids, and Related Interdisciplinary Topics, vol. 55, no. 3, pp. R2093-R2096, 1997.

[16] R. Lyons, "Asymptotic enumeration of spanning trees," Combinatorics Probability and Computing, vol. 14, no. 4, pp. 491-522, 2005.

[17] Z. Z. Zhang, S. Y. Gao, L. C. Chen, S. G. Zhou, H. J. Zhang, and J. H. Guan, "Mapping Koch curves into scale-free small-world networks," Journal of Physics A: Mathematical and Theoretical, vol. 43, no. 39, Article ID 395101, 2010.

[18] Z. Z. Zhang, H. X. Liu, B. Wu, and S. G. Zhou, "Enumeration of spanning trees in a pseudofractal scale-free web," Europhysics Letters, vol. 90, no. 6, Article ID 680029, 2010. 


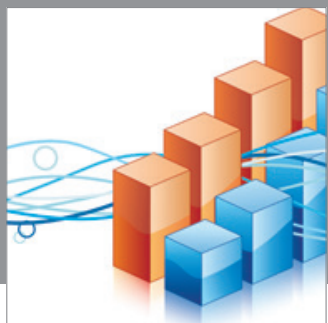

Advances in

Operations Research

mansans

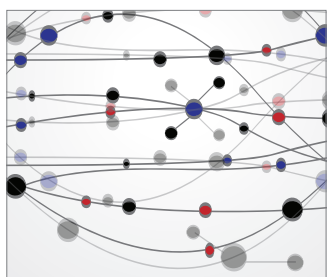

The Scientific World Journal
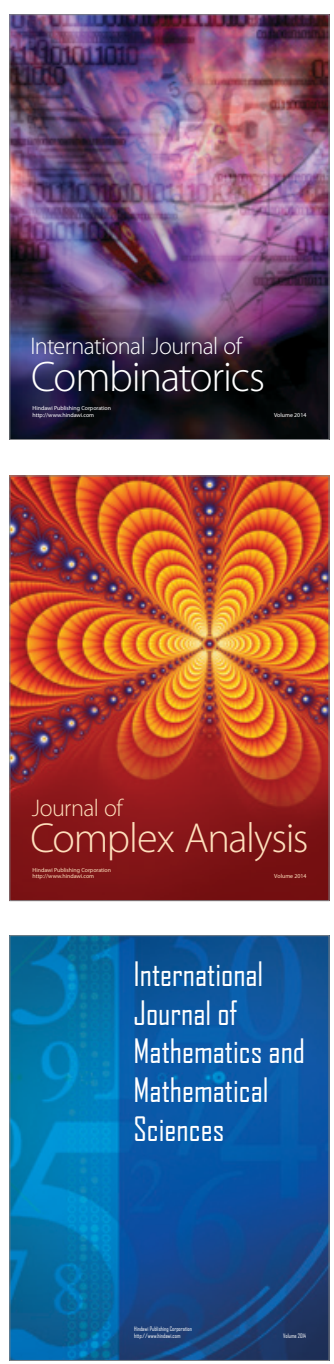
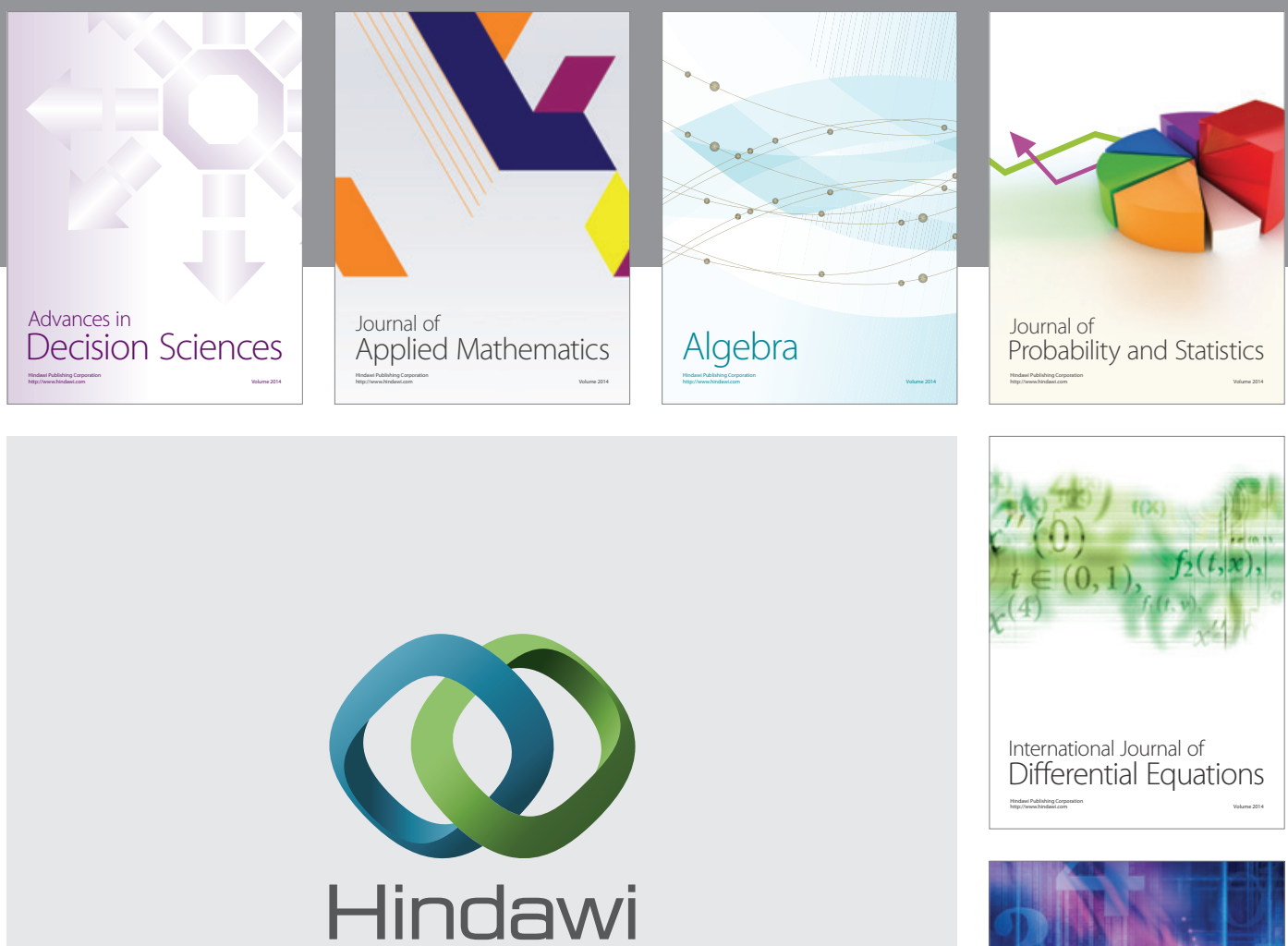

Submit your manuscripts at http://www.hindawi.com
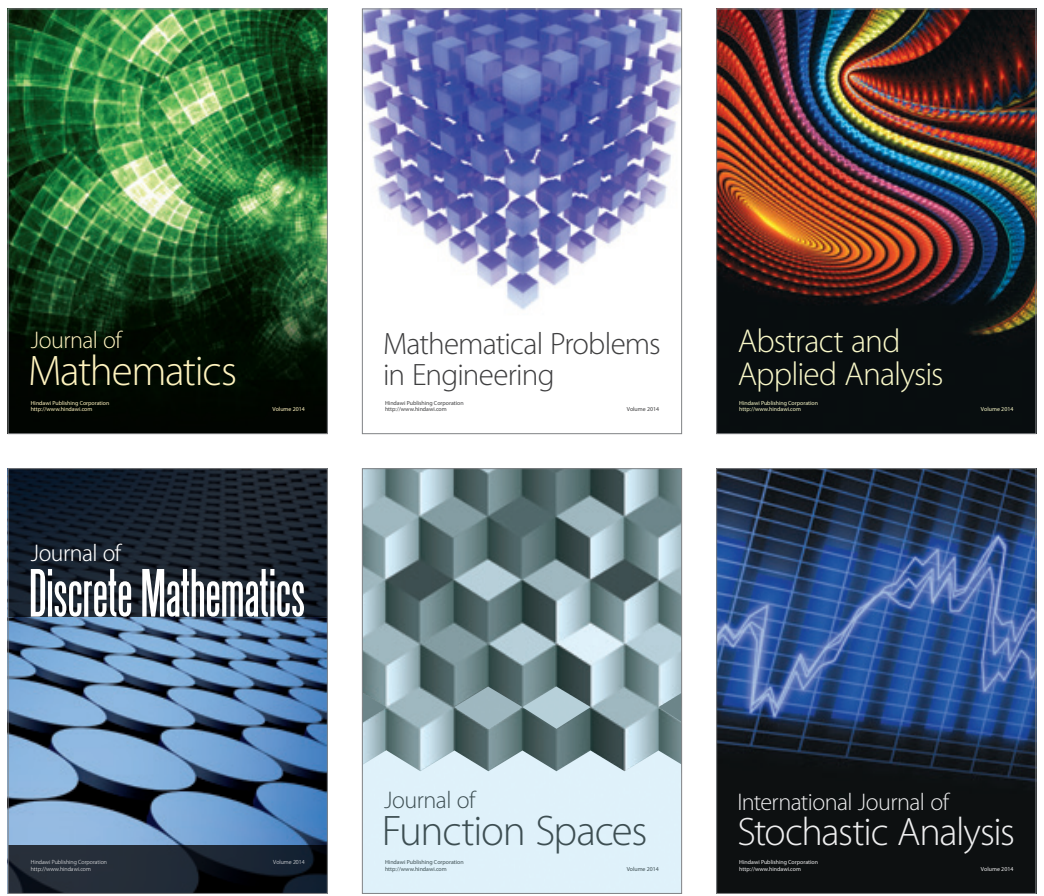

Journal of

Function Spaces

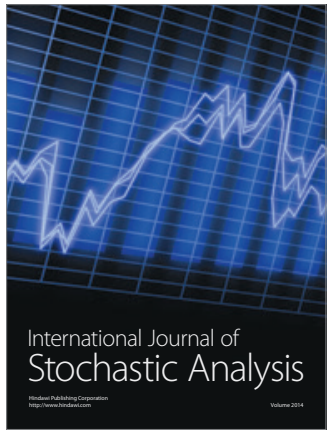

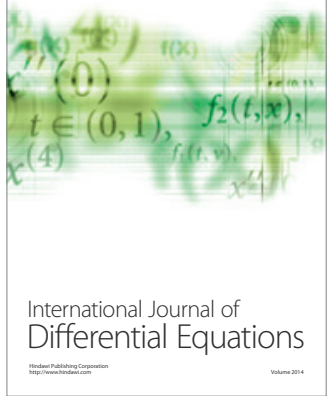
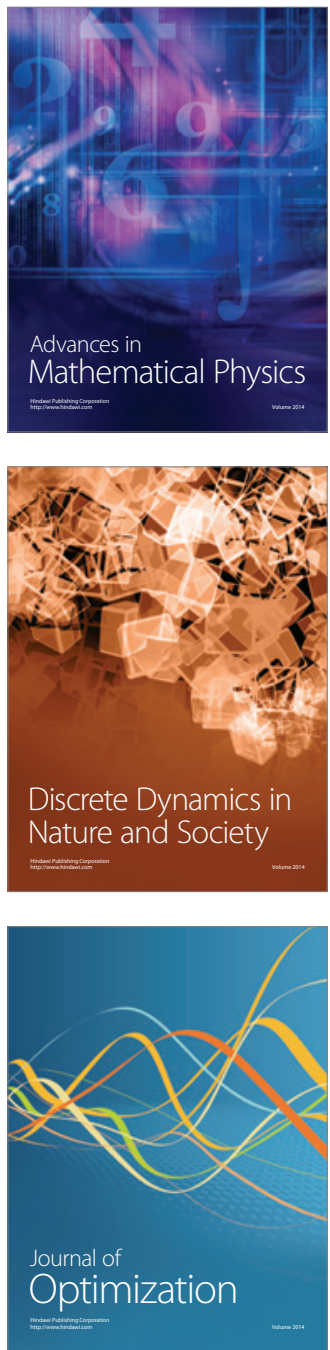